\title{
Una rigurosa investigación desde la óptica de las democracias emergentes
}

\author{
Jesús Cantú
}

JOHN M. ACKERMAN, 2007

\section{Organismos autónomos y democracia. El caso de México}

Siglo XXI, Instituto de Investigaciones Jurídicas de la Universidad Nacional Autónoma de México, México.

O on rigor metodológico, el libro establece criterios y parámetros para el análisis institucional, la evaluación de las fortalezas y debilidades de los organismos autónomos, la identificación de su impacto en la consolidación de las democracias emergentes, la demostración de la falibilidad de las tesis de los estudiosos de las ciencias políticas (basadas únicamente en la observación de las democracias consolidadas) en el contexto de dicho tipo de democracias, y los alcances y objetivos de una reforma del Estado desde la óptica de las antes dichas.

En una sólida y condensada introducción (únicamente 27 páginas) el autor analiza las argumentaciones teóricas predominantes y sus limitaciones, y plantea claramente propuestas e hipótesis alternativas. En el último párrafo de la misma establece su hipótesis: la fragmentación de la au-

\section{A Rigorous Study from the Emergent Democracies' Viewpoint}

JESÚS CANTÚ: Instituto Tecnológico y de Estudios Superiores de Monterrey, Nuevo León, México.

ice@itesm.mx

Desacatos, núm. 25, septiembre-diciembre 2007, pp. 243-248.

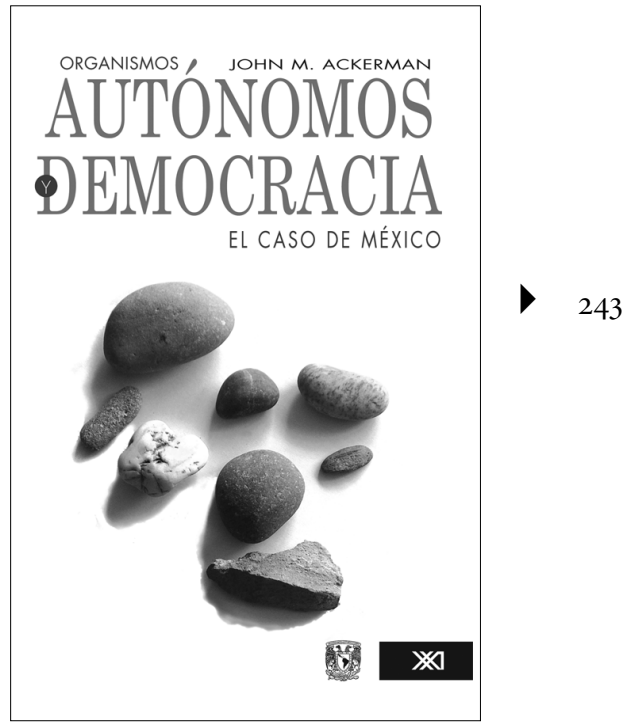

toridad y la participación de la sociedad civil son elementos que favorecen un mejor diseño y desarrollo institucional en los organismos autónomos pro-rendición de cuentas. Durante el desarrollo del libro, mediante una acuciosa documentación de las experiencias de tres organismos - Instituto Federal Electoral (IFE), Comisión Nacional de los Derechos Humanos (CNDH) y Auditoría Superior de la 


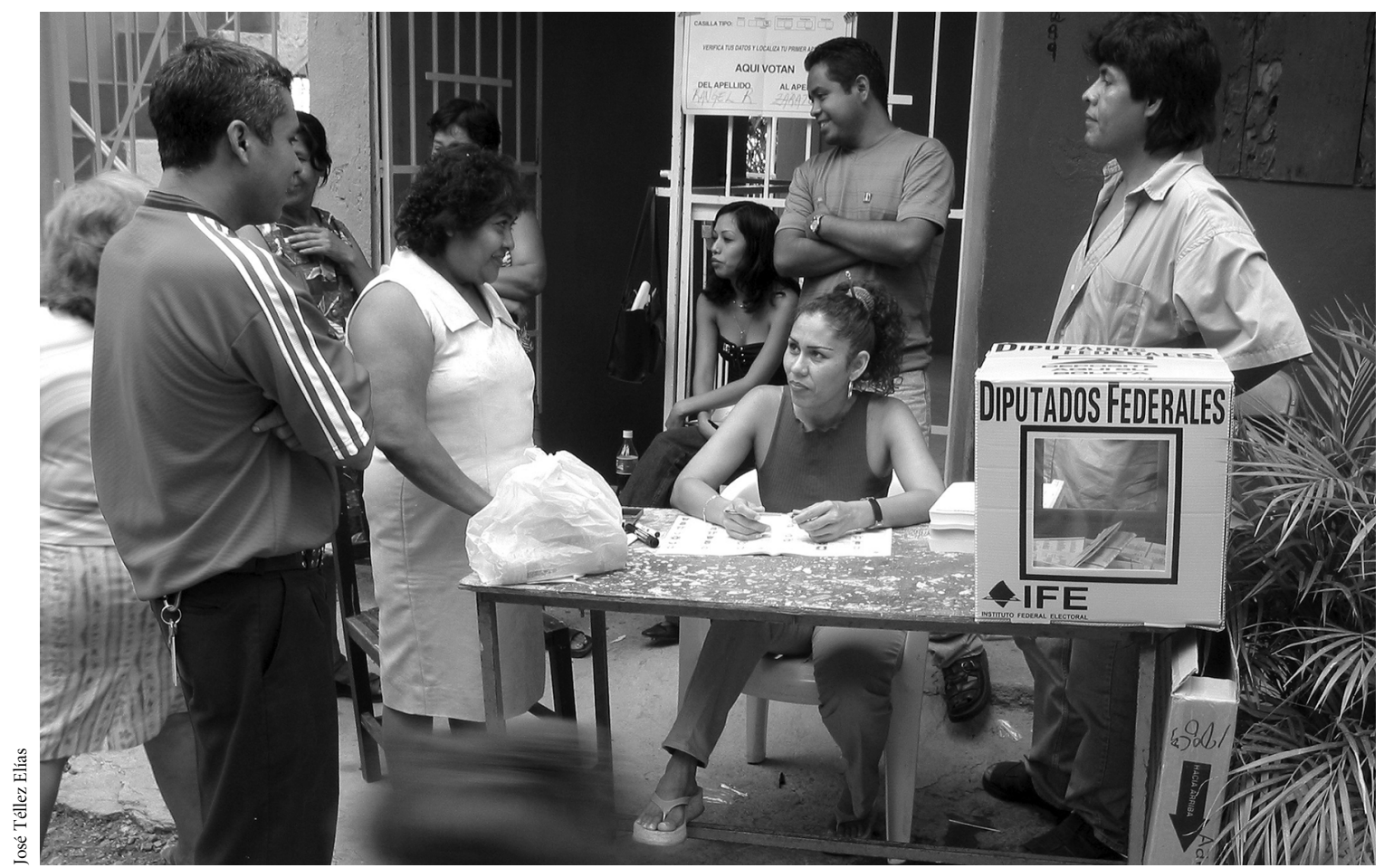

Elecciones en Acapulco, 2006.

Federación (ASF) (aunque esta última, como el mismo autor señala, no es propiamente un organismo autónomo, sí goza de autonomía técnica y de gestión)—, Ackerman demuestra que, al menos en el caso mexicano, su hipótesis se sostiene en dos aspectos: el diseño institucional y el desarrollo de las instituciones.

Respecto al análisis institucional destaca la importancia de extender dicho análisis al desempeño institucional, pues la llamada "ingeniería constitucional" enfatiza que un buen diseño facilitará buenos resultados, pero soslaya la resignificación de las reglas por los actores, el contexto político y lo que ocurre después del momento fundacional.
Para evaluar ambos aspectos establece tres dimensiones en cada uno de ellos. En el caso del diseño institucional revisa:

a) El grado de autonomía del naciente o reformado organismo; para ello repasa si goza de autonomía política, financiera, técnica y de gestión, además de los procedimientos para la designación y remoción de los titulares;

b) la capacidad, es decir, los alcances de la autoridad delegada y, particularmente, la capacidad de investigación y sanción, así como la existencia de áreas reservadas, zonas vedadas al organismo, $y$

c) la estructura, en la que engloba la correspondencia del presupuesto con las atribuciones, la existencia o no de un servicio civil de carrera, la estructura administrativa, la libertad para aprovechar fuentes externas de apoyo y trabajar con la sociedad civil.

A la par, en el desarrollo institucional incluye:

a) El mandato, que implica hasta dónde el organismo bajo la lupa persigue el cumplimiento de su mandato enérgicamente $\mathrm{o}$, al contrario, se burocratiza y simplemente sobrevive;

b) la legitimidad; aquí busca fundamentalmente el grado de confianza 
que la ciudadanía tiene en la actuación del organismo, y

c) la proactividad, que mide la capacidad del organismo para llevar el cumplimiento de sus atribuciones hasta los extremos establecidos en la ley y, en ocasiones, empujar para ensanchar dichos límites o, simplemente, limitarse a una interpretación formalista de la ley y conformarse con la lectura literal de la misma, sin correr riesgos.

Con este marco de referencia, Ackerman hace una revisión minuciosa de los tres organismos autónomos que seleccionó y cruza sus hallazgos con la estructura de la autoridad, tanto en lo referente al principal (Legislativo, Ejecutivo o ambos) en el momento de la creación o reforma del órgano, como al agente, es decir, el órgano mismo, en el momento de evaluar el desarrollo institucional. En el caso del principal revisa la coalición promulgante (fuerzas políticas participantes en la coalición que aprobó la legislación en cuestión) y establece el nivel de fragmentación de la misma (aquí incluye el número de actores que participaron en la coalición promulgante y la presencia de gobierno dividido o no); en el caso del agente, el nivel de pluralidad, formalidad y debate en el órgano de supervisión y las posibilidades de participación ciudadana en la estructura institucional. Esto último, obviamente, con la intención de probar sus hipótesis iniciales y confrontar las de los estudiosos de las ciencias sociales en función de las observaciones sobre las democracias consolidadas.
Las conclusiones a las que llega son reveladoras:

a) $\mathrm{Al}$ concluir el periodo del estudio (1990-2003) la "autonomía empoderada" (definida a partir de las tres dimensiones establecidas para medir el diseño institucional) del IFE era alta; la de la CNDH, alta-mediana; y la de la ASF, mediana.

b) Igualmente, en el desarrollo institucional el nivel del IFE era alto; el de la ASF, medio; y el de la CNDH, bajo-medio. Así, en el caso del IFE y la ASF, el nivel de desarrollo institucional corresponde al de "autonomía empoderada”; no así en el caso de la $\mathrm{CNDH}$, en la que pese a contar con una fortaleza en el diseño institucional alta-media, el desempeño institucional se encuentra muy por debajo, en el bajo-medio.

c) "[...] las coaliciones plurales y los ‘jugadores de veto’ múltiples son más efectivos que una sola autoridad unificada para la creación de estatutos e instituciones fuertes [...] Las instituciones fuertes, por lo tanto, tenderán a ser el resultado de un incremento en la pluralidad política. Esto se debe a que la pluralidad significa la inclusión de actores interesados en generar un organismo autónomo poderoso, así como un aumento en el nivel de publicidad dado a la reforma. Ambos factores hacen que el escenario de un 'sabotaje' sea más difícil." Esto lo demuestra en el hecho de que los principales avances en la "autonomía empoderada" se dan en los momentos de mayor nivel de fragmentación.

d) "[...] los organismos autónomos pro-rendición de cuentas muestran niveles más altos de desarrollo político cuando institucionalizan una diversidad de puntos de vista en su dirigencia y abren las puertas a la participación activa de la sociedad civil." En este caso, la demostración se da a partir del nivel de su "autonomía empoderada", la estructura de autoridad de los órganos y el grado de desarrollo institucional. Los que muestran un desempeño acorde con su diseño son los que cuentan con estructuras de autoridad con las características anteriores, mientras la evidencia es contraria en el que muestra un déficit de desarrollo.

Ackerman muestra las fortalezas y debilidades de cada uno de los organismos estudiados en una amplia y cuidada revisión de los momentos de creación y reforma y de la vida institucional de cada uno de ellos. Es justamente a esto a lo que le dedica la mayor parte del libro, más de 200 páginas de las 311 que lo conforman.

En el caso del IFE, el órgano que "asegura que la organización de las elecciones sea imparcial y transparente", el autor repasa las reformas constitucionales y legales de 1990, 1993, 1994 y 1996 y establece con claridad los avances reales y "cosméticos" que se lograron en cada una de ellas, así como las propuestas importantes desechadas por la coalición promulgante. Incluso analiza con detalle las condiciones políticas que acompañaban a cada uno de los procesos.

En la valoración de la importancia de las reformas al Código Federal de 
Instituciones y Procedimientos Electorales, tres aspectos en los que falta hacer más énfasis en el impacto que tienen en el diseño institucional, aunque posteriormente se valoran en el desarrollo, son:

a) La gran diferencia que establece el hecho de que la designación del coordinador de la estructura ejecutiva dependa del Consejo (secretario ejecutivo) y no del presidente del Consejo (director ejecutivo); más todavía cuando, en realidad, la dependencia era del secretario de Gobernación, quien presidía el Consejo hasta antes de la reforma de 1996. Éste, como la designación de los directores ejecutivos por parte del mismo Consejo, es un cambio que impacta directamente la estructura y, por lo mismo, aumenta la "autonomía empoderada".

b) La disposición mediante la cual la designación de los Consejos Locales ya no depende de la Junta General Ejecutiva, sino del Consejo General, pues se llegaba al absurdo de que los propios miembros de la estructura ejecutiva designaban a los integrantes del órgano ciudadano, con lo cual se perdía absolutamente el sentido de los pesos y contrapesos.

c) La transformación del Tribunal Electoral en parte de la estructura del Poder Judicial y no en un tribunal administrativo adscrito a la Secretaría de Gobernación, pues nuevamente se caía en la aberración de que los magistrados revisaban las decisiones del titular de la dependencia a la que estaban adscritos; además, con esta reforma se excluyó totalmente al Poder Ejecutivo de los órganos electorales.

\section{En el desempeño institucional el} recuento es igualmente detallado, especialmente en algunos de los asuntos más conflictivos o en los aspectos claves de la organización del proceso electoral. En lo personal, únicamente le daría todavía mayor valor — del que ya tiene en el libro- al papel que desempeñan $y$, particularmente, desempeñaron los consejeros locales y distritales en las elecciones de 1997, 2000 y 2003. Este ejército de 1992 ciudadanos y ciudadanas, que descuidaron sus labores cotidianas durante nueve meses para dedicarse a la construcción de la democracia electoral en el país, merece mayor reconocimiento, pues fueron ellos los que realmente estuvieron en el frente de batalla y desmontaron varias de las viejas prácticas del sistema electoral mexicano.

Revalorar todos estos aspectos todavía brinda más solidez a la tesis de Ackerman.

En el caso de la $\mathrm{CNDH}$, órgano "que defiende los derechos humanos de los ciudadanos de los abusos del Estado y de otros particulares", el repaso es igualmente exhaustivo en ambos aspectos. En el ámbito del diseño institucional revisa el decreto presidencial que la crea en 1990 y las legislaciones de 1992 y 1999; incluso hace un repaso detallado de las propuestas existentes por parte de las distintas fuerzas políticas de oposición.

En el desarrollo y desempeño institucional destaca la sistematización de las recomendaciones de la $\mathrm{CNDH}$, pues demuestra cómo la autonomía consti- tucional (1999) no tuvo un impacto directo en la consolidación de la institución, que fue más bien errática.

Destaca avances en dicho sentido, como las recomendaciones generales a partir del año 2001 y la atención prioritaria de la institución a la llamada "guerra sucia", el caso de "las muertas de Juárez" y la situación de los migrantes mexicanos en Estados Unidos. Sin embargo, igualmente señala los hechos de que, durante la gestión de José Luis Soberanes, que prácticamente es coincidente con la autonomía constitucional, sólo se haya emitido una recomendación a la Procuraduría General de la República; la notable disminución en el número de quejas y recomendaciones; la dispar calidad de las mismas (al mostrar incongruencias en las sanciones y no atender las verdaderas causas de los problemas); la disminución de los casos iniciados de oficio y hasta el gran déficit en la transparencia y el acceso a la información. En síntesis: "La CNDH ha dado pasos importantes, pero también ha tenido grandes tropiezos."

En el caso de la Auditoría Superior de la Federación, órgano que "en coordinación con la Cámara de Diputados vigila el buen uso del presupuesto federal y previene y sanciona la corrupción" destaca particularmente las debilidades en el diseño institucional, pues su creación finalmente fue el resultado de la alianza entre el Partido de la Revolución Institucional (PRI) y el Partido de Acción Nacional (PAN) en 1999. Resalta la fobia del diputado priísta Jorge Estefan a la participación ciudadana en la estructura de dicho órgano y en los Consejos Consultivos, 
pues el legislador consideraba que su participación "resultaría una gran contaminación". Posteriormente, éste se apropia (para los diputados) de la representación ciudadana. Dicha postura no es, definitivamente, exclusiva del citado congresista, sino extensiva a gran parte de los miembros de la clase política mexicana.

En cuanto al desempeño institucional, el autor menciona positivamente la práctica de las auditorías del desempeño y su postura en asuntos tan controvertidos y trascendentes para el país, como el caso del Fobaproa y el conflicto con la Comisión Reguladora de Energía en relación con los contratos de servicios múltiples. En este caso es, por demás, evidente el efecto dinamizador en el desarrollo institucional de la pluralidad en la Cámara de Diputados.

En general, en la "autonomía empoderada" hay dos aspectos que se deben revaluar y analizar más detenidamente: la integración del órgano de gobierno y la publicidad de sus deliberaciones, decisiones y acciones. Uno de los elementos centrales del éxito del IFE fue justamente la integración de un órgano colegiado de dirección (lo que no sucede en ninguno de las otras dos instituciones), seguido por la disposición de que todas las sesiones de dicha instancia sean públicas. Esta combinación es virtuosa, pues le da una amplia visibilidad pública y se convierte en un acicate permanente para los integrantes del mismo.

En lo que se refiere a las diferencias entre las democracias consolidadas y las emergentes, Ackerman señala particularmente dos, que impactan direc-

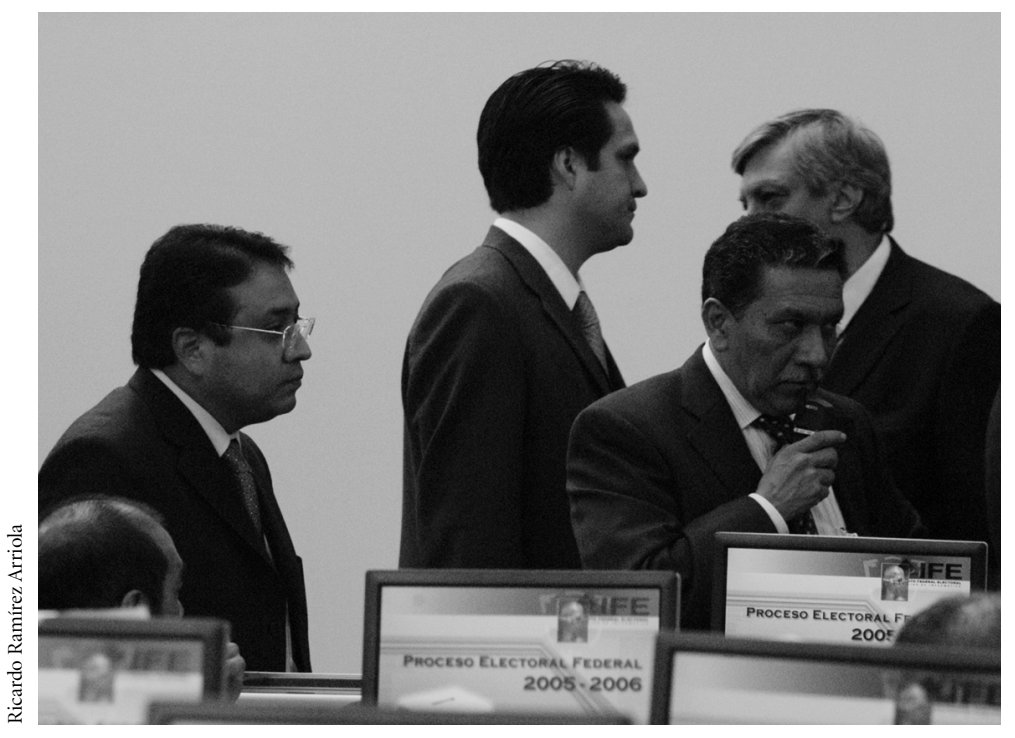

Oficinas del IFE, proceso electoral de 2006.

tamente el efecto de los “jugadores de veto" y los gobiernos divididos. La primera es que en los países en proceso de democratización "generalmente existe una fuerte influencia de la coalición autoritaria previa. Esto añade una división adicional a la estructura política [...] los países inmersos en procesos de democratización están fuertemente escindidos entre los defensores y los colaboradores del viejo régimen y los propulsores del nuevo régimen".

Con ello, al problema de arribar a acuerdos se le añade el de aprobar una legislación efectiva, que asegure una verdadera reforma institucional. Para reforzar su argumento, el autor cita a Josephine Andrews y Gabriella Montinola: "en democracias emergentes, el acuerdo político no es el único obstáculo potencial para las reformas. La tarea más importante para los reformadores es la prevención de la aprobación de legislación co- rrupta y asegurar la adecuada aplicación de las reformas genuinas. [Por lo tanto] cuando el imperio de la ley depende de la aprobación de legislación para crear y fortalecer instituciones, como poderes judiciales y agencias reguladoras independientes, así como para prevenir una legislación que debilite estas instituciones, un aumento en el número de los jugadores de veto fortalece el imperio de la ley".

La segunda diferencia la constituye la velocidad del cambio político, ya que éste es "inevitablemente más lento en una democracia antigua que en una emergente". Y esta diferencia en la velocidad conduce a dinámicas distintas en el desempeño institucional y, en ocasiones, opuestas. Por ello asienta: "mientras un incremento en la cantidad de jugadores de veto en las democracias antiguas aparentemente limita la capacidad innovadora de las políticas pública (Tsebelis, 1995, 2002), este 
estudio sugiere que el mismo incremento puede estimular la innovación en las democracias emergentes." Así refuta, al menos en el caso de México, las hipótesis tradicionales de que el estatuto de un organismo gubernamental es más fuerte cuando lo promueve una coalición unificada y que los organismos autónomos tienen un mejor desempeño cuando son dirigidos por servidores públicos altamente capacitados que se encuentran aislados de las influencias externas.

Ackerman propone en su libro profundizar y ampliar "las teorías convencionales acerca de los jugadores de veto y gobiernos divididos. Estas teorías constituyen herramientas efectivas para analizar la dinámica de elaboración de políticas públicas, pero habría que modificar sus predicciones cuando

ellas son utilizadas para analizar procesos de transición democrática”.

Respecto al ámbito del desempeño de los organismos autónomos señala: "Específicamente, la lección más importante es que los organismos autónomos son más eficaces cuando existe un debate abierto entre los integrantes de la dirección del organismo así como cuando prevalece la apertura hacia la participación de la sociedad civil. Esta transparencia, debate y participación sacuden la inercia burocrática e inoculan al organismo en contra de una gestión parcial o corrupta [...] los organismos autónomos son más exitosos cuando se encuentran sujetos a un órgano de vigilancia plural, están abiertos a la participación ciudadana y tienen un desempeño público dinámico y proactivo."

Un aspecto que no aborda en forma explícita, pero que subyace en toda su argumentación, es la cultura política prevaleciente en las democracias emergentes. Cultura política que se manifiesta en las resistencias conscientes o inconscientes al cambio y en la permanencia de prácticas autoritarias. Ésta puede abordarse desde varios aspectos en la temática que trata en el libro, pues no es casual que los tres órganos autónomos que estudia estén ligados a temas cuya expresión o vulneración se potencia en los regímenes autoritarios (elecciones, derechos humanos y corrupción) y, por lo mismo, crece con ello la demanda social de atenderlos. También sería interesante revisar las historias de vida de los protagonistas de las tres instancias para ver si pueden construirse algunas correlaciones que expliquen los comportamientos tan desiguales de los actores políticos en circunstancias similares.

En cuanto a la "consolidación democrática” y la reforma del Estado, el autor desmenuza las cuatro trampas conceptuales en las que suele caer la literatura contemporánea:

a) El minimalismo, que reduce la consolidación democrática a la democracia electoral.

b) El pensamiento teleológico, que plantea como única alternativa la construcción de instituciones al estilo de las democracias consolidadas del norte, sin tomar en cuenta que su realidad, en muchas ocasiones, difiere de su visión ideal y de las especificidades de cada nación, y de la posibilidad de desarrollar variantes más avanzadas. c) El maximalismo derrotista, que plantea una falsa dicotomía entre democracia participativa y representativa y la dependencia del Estado del capital. Antes de cualquier reforma los que postulan dicha tesis se dan por derrotados ante las fuerzas del mercado y los conflictos de clase.

d) El economicismo, según el cual la democracia y el mercado requieren de las mismas estructuras institucionales básicas para funcionar; sus adeptos consideran que la única reforma democrática del Estado posible es la reforma neoliberal.

En contrapartida, siguiendo a Johnatan Fox y Guillermo O'Donnell, Ackerman define la "reforma democrática del Estado" como "las reformas que incrementan la rendición de cuentas del aparato de Estado a la sociedad en general y que consolidan a la ciudadanía al ampliar la igualdad de oportunidades y la protección para todos los miembros de la sociedad en las diversas esferas de la vida."

Así, el libro de John Ackerman aporta herramientas para el análisis político; demuestra que no es posible extrapolar las teorías de las ciencias políticas construidas con base en el estudio de las democracias consolidadas a las democracias emergentes; establece rumbos para afrontar la "reforma democrática del Estado" desde la realidad de los países latinoamericanos y con altura de miras; genera inquietudes; $y$ abre nuevas líneas y temas de investigación.

agosto de 2007 